\title{
Anomalous Drude Model
}

\author{
Hermann Schulz-Baldes, \\ Laboratoire de Physique Quantique, Université Paul-Sabatier, 118, Route de Narbonne, 31062 Toulouse Cedex, France.
}

\begin{abstract}
A generalization of the Drude model is studied. On the one hand, the free motion of the particles is allowed to be sub- or superdiffusive; on the other hand, the distribution of the time delay between collisions is allowed to have a long tail and even a non-vanishing first moment. The collision averaged motion is either regular diffusive or Lévy-flight like. The anomalous diffusion coefficients show complex scaling laws. The conductivity can be calculated in the diffusive regime. The model is of interest for the phenomenological study of electronic transport in quasicrystals.
\end{abstract}

PACS number: 71.10.Ca, 05.60.+w, 72.10Bg

Drude's model [1,2] considers a gas of independent charged classical particles. Their ballistic motion is perturbed by collisions at random times distributed according to the Poisson process of rare events. At every collision, a particle exchanges impulsion and energy with a scatterer in a random way. The scatterers are supposed to be at thermal equilibrium and the temperature of this bath partially determines the distribution of the scattering parameters. The collisions allow the particles to dissipate impulsion and energy to the bath, but they also force the collision averaged time evolution to be diffusive. Placing the system in an exterior electric field, one obtains the celebrated Drude formula for the conductivity in this diffusive regime.

The first modification of the Drude model considers the free motion of particles between collisions. Let us suppose that it is characterized by an exponent $\sigma$ taking values in the interval $[0,1]$ and an anomalous speed $\vec{v}_{\sigma}$ such that

$$
\vec{x}(t)=\vec{x}(0)+\vec{v}_{\sigma} t^{\sigma}
$$

where $\vec{x}(t)$ is the position in euclidien physical space at time $t$ and $\vec{x}(0)$ the initial position at $t=0$. Of course, such a motion cannot be deduced from a Hamiltonian in the framework of classical mechanics, but it does mimic the anomalous quantum diffusion of a particle in a disorder or quasiperiodic on-site potential where quantum interferences play an important rôle [3]. This has been shown numerically for the Fibonacci and the Harper Hamiltonian [ [- as well as for two-dimensional models for quasicrystals [5]. Furthermore there is analytical evidence for the Fibonacci Hamiltonian [6]. If the Fermi level is in a region of localized states, $\sigma=0$ and (11) describes the hopping from one localized state to another. For the Anderson model, one expects diffusive quantum motion $(\sigma=1 / 2)$ in three dimensions and at low disorder if the Fermi energy is in the band center.
The particles undergo inelastic collisions at random times $t_{n}, n \in \mathbf{Z}$, with the moving disorder, notably phonons and other particles of the gas. The frozen potential such as impurity or quasiperiodic potential has already been taken into account and leads to the anomalous free motion (11). The direction of the velocity vector $\vec{v}_{\sigma}$ after collision is supposed to be completely random; the distribution of its modulus shall be given by the temperature $T$ of the bath composed by the scatterers. Solely the mean $C_{T, \sigma}$ of $\left|\vec{v}_{\sigma}\right|^{2}$ will be needed below. In the quantum mechanical framework, this constant can be calculated explicitly [7].

Finally, Drude's choice of the exponential law for the distribution of the time delay $s$ between collisions is replaced by any probability law, in particular those with a long tail. For sake of concreteness, let us consider the family of probability measures on $[0, \infty)$ given by

$$
p_{\mu, \tau}(s) d s=\frac{\mu}{(1+s / \tau)^{1+\mu}} \frac{d s}{\tau},
$$

with $\mu>0$ and $\tau>0$. While the exponent $\mu$ characterizes the tail of the distribution $p_{\mu, \tau}, \tau$ allows to vary the (inelastic mean) collision time, namely the first moment of $p_{\mu, \tau}$ (whenever it exists, that is $\mu>1$ ). Phenomenologically, increasing the collision time corresponds to lowering the temperature of the bath. Considering the number of collisions up to a given time $t$ gives a continuous time stochastic process with integer values, a so-called counting process. The only counting process which is stationary and has independent increments is the Poisson process [8]. Hence the counting processes defined by (2) do not have this Markov property. Note that, in particular, the probability to have no collision after time $s+s^{\prime}$ is bigger than the product of the probabilities to have none after time $s$ and none after time $s^{\prime}$. Let us finally remark that the explicit form of $p_{\mu, \tau}$ is of no importance for the results below as long as $\lim \sup _{s \rightarrow \infty} s^{1+\mu+\epsilon} p_{\mu, \tau}(s)$ is bounded for $\epsilon \leq 0$ and unbounded for $\epsilon>0$. Possible physical reasons for the choice (2) are not discussed here.

This accomplishes the presentation and motivation of the anomalous Drude model characterized by the parameters $\mu, \tau, \sigma$ and $T$. The first interesting quantities to calculate are the diffusion exponent $\eta$ and the (anomalous) diffusion coefficient $D_{\eta}(\tau)$ defined by means of the collision averaged mean square displacement:

$$
\mathbf{E}_{\mu, \tau, \sigma, T}\left((\vec{x}(t)-\vec{x}(0))^{2}\right) \underset{t \rightarrow \infty}{\approx} D_{\eta}(\tau) t^{2 \eta},
$$

where $\mathbf{E}_{\mu, \tau, \sigma, T}$ denotes the mean over all collision times and outcomes. Clearly $\eta \in[0,1]$. In order to give a 
precise mathematical meaning to the definition of $\eta$ and $D_{\eta}(\tau)$, let us introduce the Laplace transform

$$
\mathcal{L}_{\mu, \tau, \sigma, T}(\delta)=\int_{0}^{\infty} d t e^{-\delta t} \mathbf{E}_{\mu, \tau, \sigma, T}\left((\vec{x}(t)-\vec{x}(0))^{2}\right) .
$$

Then $\eta$ is defined to be half of the infimum over all real $\gamma$ such that $\lim _{\delta \rightarrow 0} \delta^{1+\gamma} \mathcal{L}_{\mu, \tau, \sigma, T}(\delta)=0$. An equivalent definition is [9]

$$
\eta=\frac{1}{2} \limsup _{t \rightarrow \infty} \frac{\log \left(\mathbf{E}_{\mu, \tau, \sigma, T}(\vec{x}(t)-\vec{x}(0))^{2}\right)}{\log (t)} .
$$

Whenever it exists, the diffusion coefficient is given by

$$
\begin{aligned}
D_{\eta}(\tau) & =\lim _{T \rightarrow \infty} \int_{0}^{T} \frac{d t}{T} \frac{\mathbf{E}_{\mu, \tau, \sigma, T}\left((\vec{x}(t)-\vec{x}(0))^{2}\right)}{t^{2 \eta}} \\
& =\lim _{\delta \rightarrow 0} \delta^{1+2 \eta} \mathcal{L}_{\mu, \tau, \sigma, T}(\delta),
\end{aligned}
$$

where the equality follows from a Tauberian lemma. The Laplace transform $\mathcal{L}_{\mu, \tau, \sigma, T}$ can be calculated explicitly:

$$
\mathcal{L}_{\mu, \tau, \sigma, T}(\delta)=\frac{2 \sigma C_{T, \sigma}}{\delta} \frac{\int_{0}^{\infty} d s p_{\mu, \tau}(s) \int_{0}^{s} d r e^{-\delta r} r^{2 \sigma-1}}{1-\int_{0}^{\infty} d s p_{\mu, \tau}(s) e^{-\delta s}} .
$$

Hence the results can be summerized by the phase diagram given in Figure 1 where $\eta$ and $D_{\eta}(\tau)$ in the regions I to $\mathbf{I V}$ are given by

$$
\begin{array}{ccc}
\text { I } & \eta=\frac{1}{2}, & D_{\eta}(\tau)=\tau^{2 \sigma-1} C_{T, \sigma} \frac{\left\langle s^{2 \sigma}\right\rangle_{\mu, 1}}{\langle s\rangle_{\mu, 1}}, \\
\text { II } & \eta=\sigma+\frac{1-\mu}{2}, & D_{\eta}(\tau)=\tau^{\mu-1} C_{T, \sigma} C_{\mu}^{\mathrm{II}}, \\
\text { III } & \eta=\mu, & D_{\eta}(\tau)=\tau^{2 \sigma-\mu} C_{T, \sigma} C_{\mu}^{\mathrm{III}}, \\
\text { IV } & \eta=\sigma, & D_{\eta}(\tau)=C_{T, \sigma} C_{\mu}^{\mathrm{IV}},
\end{array}
$$

where $\langle.\rangle_{\mu, \tau}$ denotes the mean with respect to $p_{\mu, \tau}$ and $C_{\mu}^{\mathrm{II}}, C_{\mu}^{\mathrm{III}}$ and $C_{\mu}^{\mathrm{IV}}$ are numerical constants independent of $\tau$. Note that $\eta$ is well defined and continuous on the critical lines separating the four phases, because its definition is independent of subdominant logarithmic terms. The diffusion coefficient as defined in (6), however, does not exist on these lines because of these logarithmic divergences. Nevertheless, the dependence of $D_{\eta}$ on $\tau$ varies continuously across the critical lines. Now follow comments on each of the four regions.

The diffusive regime $\mathbf{I}$ is remarkable because of the stability of diffusion exponent and coefficient upon change of the probability law for the time delays. The dependence of $D_{1 / 2}(\tau) \sim \tau^{2 \sigma-1}$ already appears in 10,11,9]. Because $D_{1 / 2}(\tau)$ is linked to the conductivity by an Einstein relation, this formula is of considerable importance when

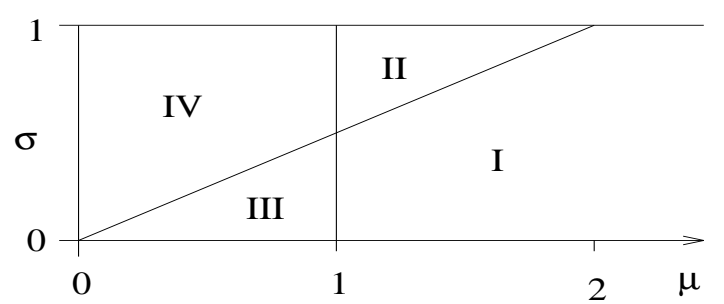

FIG. 1. Phase diagram for the anomalous Drude model

studying electronic transport in quasicrystals. These issues are further discussed below. In region II, the collision rate is not sufficient to force the collision averaged motion to be diffusive and hence it stays anomalous superdiffusive $(\eta>1 / 2)$. In literature [12,13], the motion in this regime is generally referred to as a Lévy flight and, for $\sigma=1$, the above results are known [13]. Lévy flights can either be obtained from the dynamics of a collisional model with long flying times (as above) or alternatively with long jumps (as below) (for further references, see [13,14). The latter approach can be motivated by a maximal entropy principle using Tsallis' generalized entropy [14]. Anomalous superdiffusion is of growing importance in various fields of physics such as laser cooling 15], ionic transport in oxide glasses [16], non-linear Hamiltonian dynamics [17], among others (see the review 13]). In region III, the anomalous motion is subdiffusive; the collision rate imposes the diffusion exponent and only the diffusion coefficient depends on the free motion. The case $\sigma=0$ is already studied in 18,19 . In region IV, collisions are so rare that they do not affect the anomalous free motion.

The anomalous Drude model is equivalent to a continuous time random walk 18,13. This model is defined by a probability $\psi(\vec{r}, s) d s$ that the walker (or particle) remains for a time $s$ at a given site before performing a jump of length $\vec{r}$ between $s$ and $s+d s$. If $\psi(\vec{r}, s)=\hat{\psi}(\vec{r}) \tilde{\psi}(s)$, the random walk is said to be separable. For the anomalous Drude model, one supposes that the particle stays at a given site according to (11) and that the jump length is determined by (2) according to the length of the waiting time. Hence the corresponding continuous time random walk is separable only for $\sigma=0$.

It is interesting to compare the obtained results with a corresponding discrete time random walk. In fact, as long as $\mu>1$ (regions $\mathbf{I}$ and $\mathbf{I I}$ ), the collision time $\langle s\rangle_{\mu, \tau}$ is finite. It is hence appealing to consider a random walker which makes a jump at times $N\langle s\rangle_{\mu, \tau}, N \in \mathbf{N}$, with spherical symmetric jump probability

$$
P_{\mu, \tau, \sigma, T}(\vec{x}) d^{d} x=p_{\mu, \tau}\left(\frac{|\vec{x}|^{1 / \sigma}}{C_{T, \sigma}^{1 / 2 \sigma}}\right) \frac{|\vec{x}|^{-1+1 / \sigma}}{S^{d-1} C_{T, \sigma}^{1 / 2 \sigma}} d \Omega d|\vec{x}|,
$$

where $|\vec{x}| \geq 0$ is the radial variable in spherical coordinates, $d \Omega$ the area element on the $(d-1)$-sphere and $S^{d-1}$ 
the area of the latter. Note that $P_{\mu, \tau, \sigma, T}(\vec{x}) \sim|\vec{x}|^{-1-\mu / \sigma}$ as $|\vec{x}| \rightarrow \infty$. The distribution of the position of the walker after $N$ jumps is given by the $N$-th convolution product of $P_{\mu, \tau, \sigma, T}$. The diffusion exponents $\eta^{\prime}(q)$ and anomalous diffusion coefficients $D_{\eta^{\prime}(q)}^{\prime}, q>0$, are then introduced by

$$
\int_{\mathbf{R}^{d}} d^{d} x P_{\mu, \tau, \sigma, T}^{* N}(\vec{x})|\vec{x}|^{q} \underset{N \rightarrow \infty}{\approx} D_{\eta^{\prime}(q)}^{\prime}(\tau) N^{q \eta^{\prime}(q)} .
$$

In region I, $\mu>2 \sigma$ so that the second moment of $P_{\mu, \tau, \sigma, T}$ exists. Therefore the central limit theorem implies that the $N$-th fold convolution product $P_{\mu, \tau, \sigma, T}^{* N}\left(x_{N}\right)$ with $x_{N}=x / \sqrt{N}$ converges in distribution to a Gaussian and one verifies that $\eta^{\prime}(q)=1 / 2$ independently of $q$. Of course, this confirms the stability of this regime addressed above. Moreover, the ratio of the second moment of $P_{\mu, \tau, \sigma, T}$ and the collision time $\langle s\rangle_{\mu, \tau}$ gives $D_{1 / 2}^{\prime}(\tau)$. One has $D_{1 / 2}^{\prime}(\tau)=D_{1 / 2}(\tau)$.

In region II, $P_{\mu, \tau, \sigma, T}(x) \sim|x|^{-1-\gamma}$ with $\gamma=\mu / \sigma$ such that $1<\gamma<2$. Hence Gnedenko's generalized central limit theorem 13] implies that $P_{\mu, \tau, \sigma, T}^{* N}\left(x_{N}\right)$ with the scaling $x_{N}=x / N^{\gamma}$ converges in distribution to the spherical symmetric Lévy law $L_{\gamma, \tau, T}$ which is given by its characteristic function

$$
\log \left(\hat{L}_{\gamma, \tau, T}(\vec{k})\right)=-C_{\gamma, T}|\tau \vec{k}|^{\gamma},
$$

where $C_{\gamma, T}$ is a constant depending on $\gamma$ and $T$. Going through the details, this allows to determine the diffusion exponents given by (9) to be $\eta^{\prime}(q)=\sigma / \mu$ for all $q<$ $\mu / \sigma$. For $q>\mu / \sigma$ the integral in (9) diverges for any $N$. The anomalous diffusion coefficients $D_{\eta^{\prime}(q)}^{\prime}(\tau)$ may be calculated as well.

At first sight, it seems to be paradoxical that $\eta^{\prime}(q)$ is defined only for $q<\mu / \sigma$ while $\eta$ is defined for $q=$ 2. In fact, equation (3) gives the stochastic evolution of the mean square displacement. It exists for finite times because $|\vec{x}(t)-\vec{x}(0)| \leq C t^{\sigma}$ if the distribution of the $\vec{v}_{\sigma}$ has a distribution supported in the ball of size $C$. Therefore the diffusion exponent $\eta$ is well defined. On the other hand, for the discrete time random walk with jump probability $P_{\mu, \tau, \sigma, T}$, any step can have arbitrarily big length so that the moment $q=2$ in (9) diverges for any $N$. In other words, the distribution of the particle positions after a continuous time random walk of time $N\langle s\rangle_{\mu, \tau}$ is compactly supported while that after $N$ steps distributed according to (8) is slowly decaying. Hence there is no paradox at all.

Note that nevertheless $\eta^{\prime}(q) \leq \eta$ and their values coincide only on the lines separating regime II from the others. This is due to the non-Markov property of the counting process addressed above which favors long flying times and hence big mean square displacement in (3), but has no effect in (9).

Let us now calculate the conductivity in the diffusive regime and verify the Einstein relation. The accelerated particle motion in the exterior electric field $\overrightarrow{\mathcal{E}}$ is

$$
\vec{x}(t)=\vec{x}(0)+\vec{v}_{\sigma} t^{\sigma}+q \overrightarrow{\mathcal{E}} c_{T, \sigma} t^{2 \sigma},
$$

where $q$ is the charge of particle and $c_{T, \sigma}$ is a constant depending on $\sigma$ and temperature. As (1), equation (11) does not describe a classical Hamiltonian motion, but should be seen as a modelization of the quantum motion in an exterior electric field. In fact, if the Hamiltonian quantum dynamics without electric field leads to (11), then the motion in presence of an electric field satisfies (11) in linear approximation in $\overrightarrow{\mathcal{E}}[7]$. For ballistic free motion, (11) gives the usual accelerated motion. If $\sigma=0$, (11) means that the hopping between localized states is preferably in direction of the electric field. For $\sigma=1 / 2$, the motion (11) is ballistic in presence of an electric field which allows to calculate the conductivity even if there are no collisions. In any other case $(\sigma \neq 1 / 2)$, collisions are necessary as impulsion and energy sink (for $\sigma>1 / 2$ ) or source (for $\sigma<1 / 2$ ). Strictly speaking, energy dissipation is only needed when studying thermal transport (recall that the electrical current can be calculated in the classical Lorentz model although collisions in this model are elastic).

The average speed $\langle\vec{v}\rangle_{\overrightarrow{\mathcal{E}}, \sigma, \mu, \tau, T}$ (in the usual sense) of a particle evolving between collisions with (11) is given by the long time average of $\mathbf{E}_{\mu, \tau, \sigma, T}(\vec{x}(t)-\vec{x}(0)) / t$ or equivalently by

$$
\langle\vec{v}\rangle_{\overrightarrow{\mathcal{E}}, \sigma, \mu, \tau, T}=\lim _{\delta \rightarrow 0} \delta^{2} \int_{0}^{\infty} d t e^{-\delta t} \mathbf{E}_{\mu, \tau, \sigma, T}(\vec{x}(t)-\vec{x}(0)) .
$$

For this limit to exist and to be finite, parameters have to be in the diffusive regime $\mathbf{I}$. The calculation gives $\langle\vec{v}\rangle_{\overrightarrow{\mathcal{E}}, \sigma, \mu, \tau, T}=q c_{T, \sigma} D_{1 / 2}(\tau) \overrightarrow{\mathcal{E}} / C_{T, \sigma}$. If $n$ is the particle density, the direct conductivity is thus

$$
\hat{\sigma}=\tau^{2 \sigma-1} q^{2} n c_{T, \sigma} \frac{\left\langle s^{2 \sigma}\right\rangle_{\mu, 1}}{\langle s\rangle_{\mu, 1}},
$$

and the Einstein relation reads

$$
\hat{\sigma}=\frac{q^{2} n c_{T, \sigma}}{C_{T, \sigma}} D_{1 / 2}(\tau) .
$$

The anomalous Drude formula (13) was obtained in [10,11 and later on in a purely quantum mechanical context in 9,7].

Using the physical origin of the exponent $\sigma$ as indicated above, the interpretation of (13) is the following. If $\sigma<1 / 2$, the particles are trapped by the quantum interference phenomena in the on-site potential and, in order to have appreciable conductivity, there is a need for non-elastic collisions (as in Mott's hopping conductivity). Consequently the conductivity increases with increasing collision rate (smaller $\tau$ ) and vanishes if there are no collisions $(\tau=\infty)$. On the other hand, if $\sigma>1 / 2$, the conductivity is infinite if there are no collision and it decreases with increasing collision rate because the 


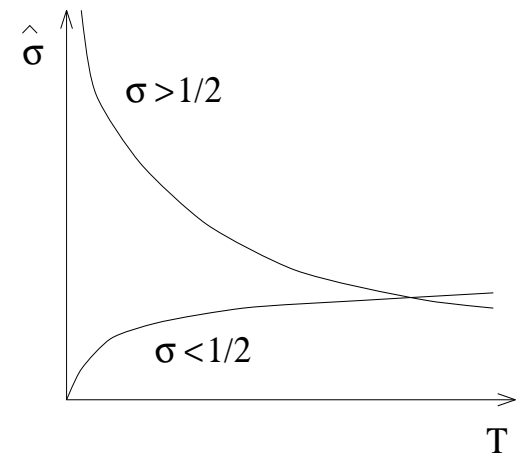

FIG. 2. Schematic representation of the temperature behavior of the conductivity as given by the anomalous Drude formula

collisions slow down the free superdiffusive motion. If $\sigma=1 / 2$, the conductivity is independent of the collision rate.

The mechanism just described is probably the physical origin for the Mooij plot of quasicrystals with differing quality. Experiment shows [20], that for those with a conductivity bigger than some critical value, the temperature derivative of the conductivity at low temperature is negative, and for those with a conductivity smaller than the critical value, the derivative is positive. For the phenomenological explanation, one supposes that $\tau \sim T^{-\alpha}$ for some $\alpha>0$. According to (13), this leads to the behavior of the conductivity given in Figure 2. Now, the higher the quality of the quasicrystal, the lower is the diffusion exponent (this expresses the continuous metalinsulator transition in quasicrystals). The combination of these two facts explains the Mooij plot; the critical value corresponds to the materials with $\sigma=1 / 2$. Other phenomena such as the inverse Mathiessen rule can be qualitatively explained by the anomalous Drude formula [7].

In summary, the anomalous Drude model modelizes the quantum motion of a particle in an on-site potential which further undergoes collisions with phonons or other particles by a stochastic process. The diffusion exponent and coefficient of the model show complex scaling laws. Furthermore the electrical conductivity can be calculated in the diffusive regime and the Einstein relation is valid.

Acknowledgement: the author would like to thank J. Bellissard, C. Sire, M. Zarraouti and R. Fleckinger for numerous discusions and comments.

[1] P. Drude, Annalen der Physik 1, 566 (1900).

[2] N.W. Ashcroft and N.D. Mermin, Solid State Physics (Saunders Co, Philadelphia, 1976).

[3] C. Sire, in Lectures on quasicrystals, edited by F. Hip- pert, D. Gratias (Les Editions de Physique, Les Ulis, 1994).

[4] H. Hiramoto and S. Abe, J. Phys. Soc. Japan 57, 230 (1988); and J. Phys. Soc. Japan 57, 1365 (1988).

[5] C. Sire, B. Passaro and V. Benza, Phys. Rev. B 46, 13751 (1992); and J. Non Crystalline Sol. $153 \& \mathbf{1 5 4}$, 420 (1993).

[6] F. Piéchon, Phys. Rev. Lett. 76, 4372 (1996).

[7] H. Schulz-Baldes and J. Bellissard, Anomalous transport: transport theory revisited, work in preparation.

[8] H. Bauer, Wahrscheinlichkeitstheorie, 4. Auflage, (de Gruyter, Berlin, New York, 1991).

[9] J. Bellissard and H. Schulz-Baldes, in Proceedings of the 5th International Conference on Quasicrystals, Editors C. Janot, R. Mosseri, World Scientific, Singapore, 1995; and Mellin transform approach to spectral and diffusion exponents, submitted to Annales de l'Institut Henri Poincaré, May 1996.

[10] C. Sire and D. Gratias, in NATO ASI Series B: Physics Vol. 319, edited by P. Turchi and A. Gonis, (Plenum Press, New York and London, 1994).

[11] D. Mayou, in Lectures on quasicrystals, edited by F. Hippert, D. Gratias (Les Editions de Physique, Les Ulis, 1994).

[12] B. Mandelbrot, The Fractal Geometry of Nature, (Freeman, San Francisco, 1982).

[13] J. P. Bouchaud and A. Georges, Phys. Rep. 195, 127 (1991).

[14] D. Zanette and P. Alemany, Phys. Rev. Lett. 75, 366 (1995).

[15] F. Bardou, J. P. Bouchaud, O. Emile, A. Aspect and C. Cohen-Tannoudji, Phys. Rev. Lett. 72, 203 (1994).

[16] D. Sidebottom, P. F. Green and R. K. Brow, Phys. Rev. B 512770 (1995).

[17] G. M Zaslavsky, D. Steven and H. Weitzner, Phys. Rev E 48, 1683 (1993).

[18] E. W. Montroll and G. H Weiss, J. Math. Phys. 6, 167 (1965).

[19] E. W. Montroll and H. Scher, J. Stat. Phys. 9, 101 (1973).

[20] C. Berger, in Lectures on quasicrystals, edited by F. Hippert, D. Gratias (Les Editions de Physique, Les Ulis, 1994). 